\title{
Predicting personality disorder functioning styles by the Chinese Adjective Descriptors of Personality: a preliminary trial in healthy people and personality disorder patients
}

\author{
Hongying Fan ${ }^{1}$, Qisha Zhu' ${ }^{1}$ Guorong Ma $^{2}$, Chanchan Shen ${ }^{1}$, Bingren Zhang ${ }^{1}$ and Wei Wang ${ }^{1,2^{*}}$
}

\begin{abstract}
Background: Cultural and personality factors might contribute to the clinical differences of psychiatric patients all over the world including China. One cultural oriented Chinese Adjective Descriptors of Personality (CADP) designed to measure normal personality traits, might be specifically associated with different personality disorder functioning styles.

Methods: We therefore have invited 201 healthy volunteers and 67 personality disorder patients to undergo CADP, the Parker Personality Measure (PERM), and the Plutchik-van Praag Depression Inventory (PVP) tests.

Results: Patients scored significantly higher on PVP scale and all 11 PERM personality disorder functioning styles, as well as CADP Emotional and Unsocial traits. The PVP was significantly correlated with some CADP traits and PERM styles in both groups. In healthy volunteers, only one CADP trait, Unsocial, prominently predicted 11 PERM styles. By contrast in patients, CADP Intelligent predicted the PERM Narcissistic and Passive-Aggressive styles; CADP Emotional the PERM Paranoid, Borderline, and Histrionic styles; CADP Conscientious the PERM Obsessive-Compulsive style; CADP Unsocial the PERM Schizotypal, Antisocial, Narcissistic, Avoidant, Dependent, and Passive-Aggressive styles; CADP Agreeable the PERM Antisocial style.

Conclusion: As a preliminary study, our results demonstrated that, in personality disorder patients, all five CADP traits were specifically associated with almost all 11 personality disorder functioning styles, indicating that CADP might be used as an aid to diagnose personality disorders in China.
\end{abstract}

Keywords: Chinese culture, Personality disorder patients, The Chinese Adjective Descriptors of Personality (CADP), The Parker Personality Measure (PERM)

Abbreviations: CADP, Chinese Adjective Descriptors of Personality; PERM, Parker personality measure; PVP, Plutchik-van Praag depression inventory; ZKPQ, Zuckerman-Kuhlman personality questionnaire.

\section{Background}

There is evidence that the psychiatric disorder patients display some clinical or epidemiological differences all over the world including China. For instance, Chinese adolescents have experienced more depression than Japanese and Korean adolescents did [1], Chinese elders

\footnotetext{
* Correspondence: drwangwei@zju.edu.cn; wangmufan@msn.com

* Correspondence: drwangwei@zju.edu.cn; wangmufan@msn.com
'Department of Clinical Psychology and Psychiatry/ School of Public Health,

* Correspondence: drwangwei@zju.edu.cn; wangmufan@msn.com
'Department of Clinical Psychology and Psychiatry/ School of Public Health,
Zhejiang University College of Medicine, Yuhangtang Road 866, Hangzhou, Zhejiang 310058, China

${ }^{2}$ Department of Psychology and Behavioral Sciences, Zhejiang University College of Sciences, Hangzhou, China
}

(c) 2016 The Author(s). Open Access This article is distributed under the terms of the Creative Commons Attribution 4.0 International License (http://creativecommons.org/licenses/by/4.0/), which permits unrestricted use, distribution, and reproduction in any medium, provided you give appropriate credit to the original author(s) and the source, provide a link to the Creative Commons license, and indicate if changes were made. The Creative Commons Public Domain Dedication waiver (http://creativecommons.org/publicdomain/zero/1.0/) applies to the data made available in this article, unless otherwise stated. have the lowest standardized prevalence of sleep comMexico, and India [2], Chinese patients with major depressive disorder have lower suicidality tendency than those in South Korea [3], and Chinese bipolar disorder patients have a remarkably lower comorbidity of alcohol problems than the Western bipolar patients did [4]. Moreover, Huang Y et al. [5] found that the prevalence of personality disorders in China were higher than that in Western Europe but lower than that in USA.

One might easily suspect that all the above-mentioned differences are associated either with the uniqueness in 
Chinese culture, or with the personality trait differences. Recently, a questionnaire based on the Chinese adjective pool, the Chinese Adjective Descriptors of Personality (CADP) [6], has been developed to measure the normal traits of Intelligent, Emotional, Conscientious, Unsocial and Agreeable, which are comparable to the traits measured by the five-factor model of personality, the Openness to Experience, Neuroticism, Conscientiousness, Extraversion, and Agreeableness respectively. Since its development is based on the Chinese culture, CADP might be more suitable to measure the personalityrelated psychiatric disorders in China. Recently, it has been demonstrated that the CADP traits were correlated respectively with the clinical symptoms of two types of bipolar disorder in Chinese culture [7].

Empirical evidence has established the relationships between normal and disordered personality traits, for instance, between the normal personality traits measured through the five-factor model such as the NEO-PI-R [8] and the clinically defined personality disorders in the general and clinical populations $[9,10]$. A recent study [11] has also shown that the alternative five-factor model of normal traits measured by the Zuckerman-Kuhlman Personality Questionnaire (ZKPQ) [12] were associated with the personality disorder functioning styles measured by the Parker Personality Measure (PERM) [13]. The question therefore arises whether the CADP normal traits are associated with the disordered personality in Chinese healthy volunteers as well as in personality disorder patients.

It has been suggested that the abnormal personalities are the extremes of normal personality variation $[14,15]$. Numerous results indicate that personalitydescriptive models such as the five-factor model are able to capture substantial variance of personality pathology in adults [16] and in adolescents [17, 18]. For instance, Openness to Experience is significantly correlated with personality disorders such as the paranoid, schizotypal [19], obsessive-compulsive, narcissistic, and antisocial personality disorders [20]. Neuroticism is correlated with the paranoid, schizotypal, antisocial, borderline, narcissistic, and obsessivecompulsive personality disorders [20]. Conscientiousness is associated with the borderline, antisocial, obsessive-compulsive [20], and passive-aggressive [21] personality disorders. Extraversion is highly consistent with the schizotypal, narcissistic, antisocial, avoidant, and passive-aggressive personality disorders [22, 23]. Agreeableness is associated with the antisocial, narcissistic, and dependent personality disorders $[8,22]$. We thus hypothesized that (1) CADP Intelligent would be correlated with the PERM Paranoid, Schizotypal, Antisocial, Narcissistic, and Obsessive-Compulsive personality disorder functioning styles; (2) Emotional, the Paranoid, Schizotypal, Antisocial, Borderline, Narcissistic, and Obsessive-Compulsive styles; (3) Conscientious, the Borderline, Antisocial, ObsessiveCompulsive and Passive-Aggressive style; (4) Unsocial, the Schizotypal, Narcissistic, Antisocial, Avoidant, and Passive-Aggressive styles; (5) Agreeable, the Antisocial, Narcissistic, and Dependent styles; and (6) these correlations would be more pronounced in personality disorder patients. We therefore have invited both healthy volunteers and personality disorder patients to undergo tests of CADP and PERM in the current study.

\section{Methods \\ Participants}

Two hundred and one healthy participants (113 women, 88 men; mean age, 20.56 years \pm 1.31 S.D., age range, 1826 years) and 67 patients with personality disorders (38 women and 29 men; mean age, $20.78 \pm 1.25$, age range, 18-24). The healthy participants were determined not suffering or had suffered from any neurological or psychiatric problems through a semi-structured interview. The semistructured interview was developed by the authors, which covered major classifications of neurological and psychiatric disorders, streamed with possible major complaints from clinical patients. Patients were firstly diagnosed through the DSM-IV-TR criteria [24] by an experienced psychiatrist (WW). Once a patient was diagnosed as suffering from a personality disorder, s/he would be handed over to other two co-authors (HF and CS) for a later confirmation using the Structured Clinical Interview for DSM-IV Axis II (SCID-II). The DSM-IV-TR diagnostic procedure was served as a participant-selection filter, and the SCID-II procedure was served as a diagnostic accuracy verification for the study design. Overall, over $90 \%$ congruency was reached between the DSM-IV-TR and SCIDII diagnostic labels to the recruited patients. Patient distributions were: 3 patients with the paranoid, 15 schizoid, 3 schizotypal, 2 antisocial, 2 borderline, 13 histrionic, 2 narcissistic, 4 avoidant, 5 dependent, 6 obsessive-compulsive, 10 passive-aggressive, 1 comorbid schizoid and histrionic, and 1 comorbid paranoid, histrionic and avoidant personality disorders. Patients were free from organic brain lesions according to recent magnetic resonance imaging or computed tomography scans, and were free from any antipsychotic drugs or alcohol for at least $72 \mathrm{~h}$ before test. No age $(t=1.20, p=.85)$, gender $\left(\chi^{2}=.005, p=.94\right)$ or education level $(t=.75, p=.46)$ differences were found between the two groups.

\section{Instruments}

Participants were asked to complete the following questionnaires in a quiet room. 
A. The Chinese Adjective Descriptors of Personality (CADP) [6] with 100 Chinese adjectives, is designed to measure the personality traits such as the Intelligent, Emotional, Conscientious, Unsocial and Agreeable (20 adjectives each). Participants were asked to complete the rating items using the Likert type scales: 1-very unlike me, 2-moderately unlike me, 3-somewhat like and unlike me, 4-moderately like me, and 5-very like me. The internal alphas of each CADP factor in the two groups were satisfactory (Table 1) as demonstrated previously $[6,7]$.

B. The Parker Personality Measure (PERM) [13], which measures 11 functioning styles of paranoid, schizoid, schizotypal, antisocial, borderline, histrionic, narcissistic, avoidant, dependent, obsessive-compulsive, and passive-aggressive personality disorders. Each PERM item has the same 5-point Likert scale as used in CADP measure. The internal alphas of each PERM scale in the two samples were satisfactory (Table 1) as reported previously $[11,24]$.

C. The Plutchik-van Praag Depression Inventory (PVP) [25] contains 34 items; each item has a three-point scale $(0,1,2)$, which corresponds to depressive tendencies. Participants have "possible depression" if they score between 20 and 25, or "depression" if they score higher than 25. The internal alpha of PVP in the present study was .89 , which was also comparable to the previous report [25].

\section{Data analyses and statistics}

Two-way ANOVA were applied to the five CADP trait or 11 PERM functioning style scores in the two groups (Group $\times$ Scale). Whenever a significant main effect was found, post-hoc analysis by the Student $t$ test was employed to evaluate between-group differences. The Student $t$ test was also employed to the mean PVP scores in the two groups. Inspired by a study [26], we applied the multiple linear regression analysis (stepwise method) to search for the relationships between the five CADP traits and the 11 PERM scales, taking CADP traits as potential predictors for the PERM scales. A p value less than .05 was considered to be significant. At the $\mathrm{p}$ level and with the present sample size (the smaller one, $n=67$ ), power to detect a raw effect (such as a scale score difference) was $99.3 \%$, a column effect $73.4 \%$, and an interaction effect $99.3 \%$.

\section{Results}

In both groups, the internal alphas of the five CADP traits and the 11 PERM functioning styles were satisfactory (Table 1). The mean PVP score in personality disorders $(18.25 \pm 10.97$ S.D.) was higher than that in healthy

Table 1 Internal alphas and scale scores (Mean \pm SD) of Chinese Adjective Descriptors of Personality (CADP) and the Parker Personality Measure (PERM)

\begin{tabular}{|c|c|c|c|c|c|}
\hline & \multicolumn{2}{|c|}{ Healthy controls $(n=201)$} & \multicolumn{2}{|c|}{ Personality disorders $(n=67)$} & \multirow{2}{*}{$\begin{array}{l}95 \% \\
\text { Confidence } \\
\text { Interval }\end{array}$} \\
\hline & Alpha & Score & Alpha & Score & \\
\hline \multicolumn{6}{|l|}{ CADP } \\
\hline Intelligent & .96 & $63.91 \pm 13.55$ & .96 & $60.12 \pm 16.90$ & $-7.81 \sim .224$ \\
\hline Emotional & .92 & $51.11 \pm 14.32$ & .92 & $61.93 \pm 15.98^{*}$ & $6.46 \sim 15.17$ \\
\hline Conscientious & .95 & $72.08 \pm 13.50$ & .93 & $69.49 \pm 14.46$ & $-6.40 \sim 1.23$ \\
\hline Unsocial & .90 & $36.79 \pm 10.58$ & .88 & $48.72 \pm 13.30^{*}$ & $8.38 \sim 15.48$ \\
\hline Agreeable & .91 & $74.85 \pm 11.29$ & .88 & $74.93 \pm 11.44$ & $-3.07 \sim 3.23$ \\
\hline \multicolumn{6}{|l|}{ PERM } \\
\hline Paranoid & .81 & $20.06 \pm 6.14$ & .82 & $27.39 \pm 7.87^{*}$ & $5.23 \sim 9.42$ \\
\hline Schizoid & .53 & $19.63 \pm 4.14$ & .59 & $22.48 \pm 5.41^{*}$ & $1.42 \sim 4.29$ \\
\hline Schizotypal & .67 & $9.46 \pm 3.30$ & .66 & $13.69 \pm 4.44^{*}$ & $3.06 \sim 5.40$ \\
\hline Antisocial & .74 & $18.84 \pm 5.28$ & .73 & $23.84 \pm 6.56^{*}$ & $3.25 \sim 6.75$ \\
\hline Borderline & .77 & $20.34 \pm 5.91$ & .75 & $28.57 \pm 7.51^{*}$ & $6.23 \sim 10.23$ \\
\hline Histrionic & .61 & $11.61 \pm 3.44$ & .58 & $16.16 \pm 4.02^{*}$ & $3.47 \sim 5.64$ \\
\hline Narcissistic & .78 & $15.53 \pm 4.95$ & .63 & $20.30 \pm 5.15^{*}$ & $3.35 \sim 6.20$ \\
\hline Avoidant & .78 & $24.00 \pm 6.15$ & .71 & $31.75 \pm 6.84^{*}$ & $5.88 \sim 9.62$ \\
\hline Dependent & .73 & $21.26 \pm 5.57$ & .73 & $27.30 \pm 7.19^{*}$ & $4.13 \sim 7.95$ \\
\hline Obsessive-Compulsive & .68 & $16.07 \pm 4.36$ & .61 & $20.00 \pm 4.24^{*}$ & $2.73 \sim 5.12$ \\
\hline Passive-Aggressive & .70 & $19.87 \pm 5.20$ & .76 & $23.99 \pm 6.79^{*}$ & $2.31 \sim 5.92$ \\
\hline
\end{tabular}


controls (7.96 \pm 5.55 S.D., $p<.001,95 \%$ Confidence Interval (CI): $7.51 \sim 13.07)$.

Two-way ANOVA also has detected significant differences of the five CADP traits in the two groups (group effect, F $[1,266]=11.87, p=.001$; mean square effect $(\mathrm{MSE})=2717.17$; scale effect, $F[4,1064]=$ $200.76, \quad p<.001 ; \quad$ MSE $=32253.45 ;$ and group $\times$ scale interaction effect, $\mathrm{F}[4,1064]=17.69, p<.001 ; \mathrm{MSE}=$ 2841.60). The post-hoc Student $t$ test has detected that personality disorders scored significantly higher than the controls did on the Emotional $(p<.001)$ and Unsocial $(p<.001)$ traits (Table 1$)$.

Meanwhile, two-way ANOVA has detected that there were significant differences of the 11 PERM styles between the two groups (group effect, F [1, 266] $=105.22, p<.001 ; \mathrm{MSE}=15786.73$; scale effect, $\mathrm{F}[10$, $2660]=287.45, p<.001 ; \mathrm{MSE}=4701.11 ;$ and group $\times$ scale interaction effect, $\mathrm{F}[10,2660]=9.39, p<.001$; MSE $=153.58$ ). The post-hoc Student $t$ test has detected that personality disorders scored significantly higher than the controls did on the Paranoid, Schizoid, Schizotypal, Antisocial, Borderline, Histrionic, Narcissistic, Avoidant, Dependent, Obsessive-Compulsive, and Passive-Aggressive (all ps $<.001$ ) styles (also see Table 1).

In the healthy controls, PVP was significantly correlated with CADP Intelligent $(r=-.22, p=.002)$ and Unsocial $(r=.23, p=.001)$ traits, and with PERM Paranoid $(r=.21, p=.003)$, Schizotypal $(r=.17, p=.014)$, Borderline $(r=.31, p<.001)$, Histrionic $(r=.17, p=.017)$, Avoidant $(r=.28, p<.001)$, Dependent $(r=.28, p<.001)$, and Obsessive-Compulsive $(r=.17, p=.014)$ functioning styles. In personality disorders, PVP was significantly correlated with CADP Intelligent $(r=-.40, p=.001)$ trait, and with PERM Schizotypal $(r=.35, p=.004)$, Borderline $(r=.38, p=.002)$, and Avoidant $(r=.29, p=.016)$ functioning styles.

Considering the prediction of PERM functioning styles by the CADP traits, the accounted variances (adjusted $R^{2}$ values) were ranged from .19 to .49 in controls, and from .05 to .47 in personality disorders (Table 2). In personality disorders, all predictors of a given personality disorder functioning style were prominent $(\beta s>30)$ : Intelligent predicted the Narcissistic and Passive-Aggressive styles; Emotional the Paranoid, Borderline, and Histrionic styles; Conscientious the Obsessive-Compulsive style; Unsocial the Schizotypal, Antisocial, Narcissistic, Avoidant, Dependent, and Passive-Aggressive styles; Agreeable the Antisocial style. In controls, Unsocial (the $\beta s>.30$ ) was the most prominent predictor of all the 11 personality disorder styles. For instance, Unsocial predicted the Paranoid ( $\beta$ $=.53)$, Schizoid (.52), Schizotypal (.65), Antisocial (.45), Borderline (.54), Histrionic (.47), Narcissistic (.54),
Avoidant (.60), Dependent (.54), Obsessive-Compulsive (.30) and Passive-Aggressive (.45) styles.

\section{Discussion}

This study explored the potential predictability of the CADP traits to the personality disorder functioning styles both in personality disorder patients and in healthy volunteers. The mean PVP score in personality disorders was higher than in healthy controls. Personality disorder patients scored significantly higher than the healthy volunteers did on the CADP Emotional and Unsocial traits and on all 11 PERM functioning styles. PVP was significantly correlated with CADP Intelligent (-) and Unsocial traits, and with PERM Paranoid, Borderline, Schizotypal, Histrionic, Avoidant, Dependent, and Obsessive-Compulsive functioning styles in healthy controls; while in personality disorders, it was significantly correlated with CADP Intelligent (-), and with PERM Schizotypal, Borderline, and Avoidant functioning styles. In personality disorder patients, all five CADP predictors of a personality disorder functioning style were prominent, which confirmed most of our hypotheses.

Patients scored significantly higher in PVP than the healthy volunteers did, which was in accordance with previous reports $[27,28]$. The PVP was also correlated with PERM Avoidant in our patients, which was in line with the high co-morbidity between the avoidant personality disorder and the major depressive disorder in clinics [29-31]. In our healthy volunteers, PVP was correlated with Unsocial, which agreed with the notion that the Unsocial represents the negative poles of extraversion [32] or Social Life [33], and that extraversion was significant-negatively correlated with depression [34]. In our healthy participants, PVP was also correlated with PERM Paranoid and Obsessive-Compulsive functioning styles. Indeed, the major depressive disorder was frequently co-morbid with the paranoid and obsessivecompulsive personality disorders [35].

The Emotional and Unsocial were significantly elevated in our patients, which were in line with the wellestablished trends that some personality disorders such as the borderline, avoidant, and dependent types displayed higher neuroticism [36], and that some personality disorders such as the narcissistic and histrionic types had lower extraversion [9]. All PERM styles were elevated in our patients, which were in accordance with previous results in personality disorder patients [11].

The interesting results in our two groups also pointed to the predicting PERM styles with CADP traits. Firstly, the Paranoid, Borderline, and Histrionic styles were strongly associated with the Emotional in patients. As for other researches (e.g. using NEO-PI-R or ZKPQ), neuroticism was consistently high-correlated with the paranoid and borderline personality disorders [37, 38], 
Table 2 Stepwise multiple regressions predicting the Parker Personality Measure styles with the Chinese Adjective Descriptors of Personality traits in the healthy controls and the personality disorders

\begin{tabular}{|c|c|c|c|c|}
\hline & \multicolumn{2}{|c|}{ Healthy controls $(n=201)$} & \multicolumn{2}{|c|}{ Personality disorders $(n=67)$} \\
\hline & Adjusted $R^{2}$ & $\beta(B, S E)$, predictors & Adjusted $R^{2}$ & $\beta(B, S E)$, predictors \\
\hline \multirow[t]{3}{*}{ Paranoid } & .34 & $.53(.31, .04)$ Unsocial $^{* *}$ & .47 & $.47(.23, .05)$ Emotional ${ }^{* *}$ \\
\hline & & $.14(.06, .03)$ Emotional* & & $-.25(-.17, .07)$ Agreeable* \\
\hline & & & & $.21(.12, .06)$ Unsocial* $^{*}$ \\
\hline \multirow[t]{2}{*}{ Schizoid } & .23 & $.52(.20, .03)$ Unsocial $^{* *}$ & .05 & $-.26(-.09, .04)$ Emotional $^{*}$ \\
\hline & & $-.15(-.04, .02)$ Emotional $^{*}$ & & \\
\hline \multirow[t]{2}{*}{ Schizotypal } & .41 & $.65(.20, .02)$ Unsocial** & .26 & $.38(.13, .04)$ Unsocial ${ }^{* *}$ \\
\hline & & & & $.27(.07, .03)$ Emotional $^{*}$ \\
\hline \multirow[t]{5}{*}{ Antisocial } & .49 & $.45(.23, .03)$ Unsocial** & .39 & $.45(.22, .05)$ Unsocial ${ }^{* *}$ \\
\hline & & $-.28(-.13, .03)$ Agreeable $e^{* *}$ & & $-.37(-.21, .06)$ Agreeable** \\
\hline & & $.33(.13, .02)$ Intelligent ${ }^{* *}$ & & $.29(.11, .04)$ Intelligent ${ }^{* *}$ \\
\hline & & $-.25(-.10, .03)$ Conscientious ${ }^{* *}$ & & \\
\hline & & $.16(.06, .02)$ Emotional** & & \\
\hline \multirow[t]{3}{*}{ Borderline } & .43 & $.54(.30, .03)$ Unsocial $^{* *}$ & .26 & $.52(.25, .05)$ Emotional ${ }^{* *}$ \\
\hline & & $.17(.07, .02)$ Emotional** & & \\
\hline & & $-.14(-.07, .03)$ Intelligent* & & \\
\hline \multirow[t]{2}{*}{ Histrionic } & .32 & $.47(.15, .02)$ Unsocial $^{* *}$ & .18 & $.44(.11, .03)$ Emotional** \\
\hline & & $.21(.05, .02)$ Emotional** & & \\
\hline \multirow[t]{3}{*}{ Narcissistic } & .38 & $.54(.25, .03)$ Unsocial $^{* *}$ & .14 & $.35(.11, .04)$ Intelligent ${ }^{* *}$ \\
\hline & & $.36(.13, .02)$ Intelligent ${ }^{* *}$ & & $.34(.13, .05)$ Unsocial $^{* *}$ \\
\hline & & $-.26(-.12, .03)$ Agreeable ${ }^{* *}$ & & \\
\hline Avoidant & .36 & $.60(.35, .03)$ Unsocial $^{* *}$ & .17 & $.43(.22, .06)$ Unsocial $^{* *}$ \\
\hline \multirow[t]{2}{*}{ Dependent } & .39 & $.54(.29, .03)$ Unsocial** & .15 & $.36(.22, .06)$ Unsocial ${ }^{* *}$ \\
\hline & & $-.22(-.09, .02)$ Conscientious ${ }^{* *}$ & & \\
\hline \multirow[t]{3}{*}{ Obsessive-Compulsive } & .19 & $.48(.16, .03)$ Conscientious** & .24 & $.57(.17, .04)$ Conscientious ${ }^{* *}$ \\
\hline & & $.30(.13, .03)$ Unsocial ${ }^{* *}$ & & $-.25(-.06, .03)$ Intelligent* \\
\hline & & $-.20(-.08, .03)$ Agreeable* $^{*}$ & & \\
\hline \multirow[t]{4}{*}{ Passive-Aggressive } & .36 & $.45(.37, .06)$ Unsocial ${ }^{* *}$ & .21 & $.43(.22, .06)$ Unsocial $^{* *}$ \\
\hline & & $-.25(-.11, .04)$ Agreeable* & & $.36(.15, .05)$ Intelligent ${ }^{* *}$ \\
\hline & & $.24(.09, .03)$ Intelligent* & & $-.25(-.12, .06)$ Conscientious* \\
\hline & & $-.22(-.09, .03)$ Conscientious ${ }^{* *}$ & & \\
\hline
\end{tabular}

Note: ${ }^{*} p<.05,{ }^{* *} p<.01 ; \beta s$ valued larger than .30 were bolded for clarity; $B$ and standardized error (SE) were unstandardized coefficients

and sometimes correlated with the histrionic personality disorder [39]. On the other hand, patients with paranoid, borderline, or histrionic personality disorder have less emotional understanding (i.e., emotional clarity), more difficulty to regulate their emotional states (i.e., emotional repair) and their emotional self-insights [40].

Secondly, the Schizotypal, Antisocial, Narcissistic, Avoidant, Dependent, and Passive-Aggressive personality disorder functioning styles were strongly predicted by the Unsocial trait in patients. Similarly, a relationship between the extraversion (negative pole of Unsocial) and the schizotypal (-), antisocial (-), narcissistic, avoidant (-), and dependent (-) personality disorders was reported in the healthy and patient samples $[41,42]$. The direct explanation for the association between the Passive-Aggressive style and the Unsocial trait found in patients is still lacking, however, some evidence has shown that the avoidant [38] and passive-aggressive personality disorders were associated with neuroticism-anxiety [37]. Aluja et al. [38] found the correlations between most facets of extraversion and the paranoid (-) and obsessivecompulsive (-) types were rather weak, which worked in concert with the current results, except histrionic. In clinics, the histrionic personality disorder is characterized by an excessive emotionality and attention 
seeking [24], which contrasts with the Unsocial trait that is characterized by the stupid, dull or inflexible behavior [6].

Thirdly, the Narcissistic and Passive-Aggressive styles were strongly predicted by the Intelligent trait in patients. In one study with healthy students, the Narcissistic, Antisocial and Passive-Aggressive styles were co-loaded on one broad antisocial domain [43]. Although the association between the Intelligent trait and the Antisocial style was weaker than in other styles, in Chinese culture, the antisocial personality trait covers one part of fierce and malicious, resembling the Machiavellian attitude [44]. The latter was correlated with the intelligence in western culture [45].

Fourthly, the Obsessive-Compulsive style was strongly predicted by the Conscientious trait in patients. It has been shown that the conscientiousness predicted workaholic characteristics [46], and workaholics scored higher on obsessive-compulsive personality disorder than healthy participants did [47]. Moreover, the obsessive-compulsive personality disorder presents a maladaptive variant of normal-range conscientiousness $[48,49]$.

Lastly, the Antisocial (-) style was strongly predicted by the Agreeable trait together with Unsocial in patients. A recent study has shown that the Agreeableness in the Five-Factor Nonverbal Personality Questionnaire [50] also strongly predicted the Antisocial (-) style [51]. Meantime, the Agreeable trait showed its capability of predicting the Antisocial style in our healthy participants. These associations again support that the agreeableness and antisocial traits are regarded as two opposite poles of a personality domain in both clinical and normal populations [52, 53].

One has to acknowledge that the current study is a preliminary design and suffers at least three limitations. Firstly, subsample sizes of individual personality disorders were relatively small and not equally distributed in number and in age, a larger recruitment of patients would make the current relationship clearer. Secondly, the internal reliability of the PERM Schizoid style was lower as usual, which might also influence the current results. Thirdly, we did not include a group of non-Chinese people, which would be a nice control for the current results. Fourthly, the participants were young people aged 18-26 years, and the results may not be generalizable to other age populations. Nevertheless, our study has demonstrated that all five CADP traits were correlated with almost all 11 PERM styles, and the associations in patients were more specific and clearer. Therefore our study indicates a validity of
CADP for predicting personality disorder types, and warrants its application as an aid to diagnose personality disorders in China.

\section{Conclusions}

Our preliminary study has demonstrated that, in personality disorder patients, all five CADP traits were specifically associated with almost all 11 PERM styles, and the associations in patients were specific and clear. Therefore, the validity of CADP for predicting personality disorder types indicates that CADP might be used as an aid to diagnose personality disorders in China.

\section{Funding}

The study was supported by a grant from the Natural Science Foundation of China (No. 91132715) to the correspondent author (Dr. W Wang).

Availability of data and materials

Data will be shared upon request.

\section{Authors' contributions}

WW conceived the study, HF, QZ, GM, CS, and BZ collected the data, HF, QZ, and GM analyzed the data, HF and WW drafted the paper. All authors read and approved the final manuscript.

\section{Competing interests}

The authors declare that they have no competing interests.

\section{Consent for publication}

Not applicable.

\section{Ethics approval and consent to participate}

The study was approved by the Ethics Committee of Zhejiang University College of Medicine (No. ZGL201307-2-2), and all participants gave their written informed consent to participate. The study conformed to the Helsinki Declaration concerning human rights and informed consent and followed correct procedures concerning treatment of humans in research.

Received: 15 April 2016 Accepted: 25 August 2016

Published online: 30 August 2016

\section{References}

1. Houri D, Nam EW, Choe EH, Min LZ, Matsumoto K. The mental health of adolescent school children: a comparison among Japan, Korea, and China. Glob Health Promot. 2012;19(3):32-41.

2. Mazzotti DR, Guindalini C, Sosa AL, Ferri CP, Tufik S. Prevalence and correlates for sleep complaints in older adults in low and middle income countries: a 10/ 66 Dementia Research Group study. Sleep Med. 2012;13(6):697-702.

3. Lim AY, Lee AR, Hatim A, Tian-Mei S, Liu CY, Jeon HJ, et al. Clinical and sociodemographic correlates of suicidality in patients with major depressive disorder from six Asian countries. BMC Psychiatry. 2014;14:37.

4. Tsai SY, Chen CC, Yeh EK. Alcohol problems and long-term psychosocial outcome in Chinese patients with bipolar disorder. J Affect Disord. 1997:46(2):143-50.

5. Huang $Y$, Kotov R, de Girolamo G, Preti A, Angermeyer M, Benjet $C$, et al. DSM-IV personality disorders in the WHO World Mental Health Surveys. Br J Psychiatry. 2009;195(1):46-53

6. Yu S, Wei L, He W, Chai H, Wang D, Chen W, et al. Description of personality traits by Chinese adjectives: a trial on university students. Psychol Lang Commun. 2009;13(2):5-20.

7. Yu E, Li H, Fan H, Gao Q, Tan Y, Lou J, et al. Relationship between the Chinese Adjective Descriptors of Personality and the emotional symptoms in Chinese young patients with bipolar disorders. J Int Med Res. 2015;43(6): 790-801.

8. Costa Jr PT, McCrae RR. "Normal" personality assessment in clinical practice: the NEO Personality Inventory. Psychol Assess. 1992;4(1):5-13. 
9. Saulsman LM, Page AC. The five-factor model and personality disorder empirical literature: a meta-analytic review. Clin Psychol Rev. 2004;23(8):1055-85.

10. Samuel DB, Widiger TA. A meta-analytic review of the relationships between the five-factor model and DSM-IV-TR personality disorders: a facet level analysis. Clin Psychol Rev. 2008;28(8):1326-42.

11. Huang J, He W, Chen W, Shen M, Wang W. The Zuckerman-Kuhlman Personality Questionnaire predicts functioning styles of personality disorder: a trial in healthy subjects and personality-disorder patients. Psychiatry Res. 2011;186(2-3):320-5.

12. Zuckerman M, Kuhlman DM, Joireman J, Teta P, Kraft M. A comparison of three structural models for personality: The Big Three, the Big Five, and the Alternative Five. J Pers Soc Psychol. 1993;65(4):757-68.

13. Parker $G$, Hadzi-Pavlovic D. A question of style: refining the dimensions of personality disorders style. J Pers Disord. 2001;15(4):300-18.

14. O'Connor BP, Dyce JA. Rigid and extreme: a geometric representation of personality disorders in five-factor model space. J Pers Soc Psychol. 2001;81(6):1119-30.

15. Miller JD, Lynam DR, Widiger TA, Leukefeld C. Personality disorders as extreme variants of common personality dimensions: can the Five-Factor Model adequately represent psychopathy? J Pers. 2001;69(2):253-76.

16. Costa Jr PT, Widiger TA. Personality disorders and the Five-Factor Model of personality. Washington, DC: American Psychological Association; 2002.

17. De Clercq B, De Fruyt F. Personality disorder symptoms in adolescence: a five-factor model perspective. J Pers Disord. 2003;17(4):269-92.

18. Mervielde I, De Clercq B, De Fruyt F, Van Leeuwen K. Temperament, personality, and developmental psychopathology as childhood antecedents of personality disorders. J Pers Disord. 2005;19(2):171-201.

19. Morey LC, Gunderson J, Quigley BD, Lyons M. Dimensions and categories: the "big five" factors and the DSM personality disorders. Assessment 2000;7(3):203-16

20. Costa Jr PT, McCrae RR. Personality disorders and the five-factor model of personality. J Pers Disord. 1990;4:362-71.

21. Wiggins JS, Pincus AL. Conceptions of personality disorders and dimensions of personality. Psychol Assess. 1989;1(4):305-16.

22. Widiger TA, Trull TJ. Personality and psychopathology: an application of the five-factor model. J Pers. 1992;60(2):363-93.

23. Widiger TA, Costa Jr PT, Gore WL, Crego C. Five factor model personality disorder research. In: Widiger TA, Costa PT, editors. Personality Disorders and the Five-Factor Model of Personality. 3rd ed. Washington, DC: American Psychological Association; 2013. p. 75-100.

24. American Psychiatric Association. Diagnostic and Statistical Manual of Mental Disorders, text revision. 4th ed. Washington, DC: American Psychiatric Association; 2000.

25. Plutchik $R$, van Praag HM. Interconvertibility of five self-reported measures of depression. Psychiatr Res. 1987;22(3):243-56.

26. Schrijvers DL, De Bruijn ER, Destoop M, Hulstijn W, Sabbe BG. The impact of perfectionism and anxiety traits on action monitoring in major depressive disorder. J Neural Transm. 2010;117(7):869-80.

27. Charney DS, Nelson JC, Quinlan DM. Personality traits and disorder in depression. Am J Psychiatry. 1981;138(12):1601-4.

28. Molinari V, Marmion J. Relationship between affective-disorders and Axis-॥ diagnoses in geropsychiatric patients. J Geriatr Psychiatry Neurol. 1995;8:61-4.

29. Shea MT, Glass DR, Pilkonis PA, Watkins J, Docherty JP. Frequency and implications of personality disorders in a sample of depressed outpatients. J Pers Disord. 1987;1:27-42.

30. Sanderson WC, Wetzler S, Beck AT, Betz F. Prevalence of personality disorders among patients with major depression and dysthymia. Psychiatry Res. 1992;42:93-9.

31. Pepper CM, Klein DN, Anderson RL, Riso LP, Ouimette PC, Lizardi H. DSM-III-R axis II comorbidity in dysthymia and major depression. Am J Psychiatry. 1995;152(2):239-47.

32. Costa Jr PT, McCrae RR. NEO Personality Inventory-Revised (NEO-PI-R) manual. Odessa: Psychological Assessment Resources; 1994.

33. Rudowicz E, Yue X. Compatibility of Chinese and creative personalities. Creat Res J. 2002;14:387-94.

34. Cheng $H$, Furnham A. Personality, self-esteem, and demographic predictions of happiness and depression. Pers Individ Dif. 2003;34(6):921-42.

35. Fava M, Farabaugh AH, Sickinger AH, Wright E, Alpert JE, Sonawalla S, et al. Personality disorders and depression. Psychol Med. 2002;32(6):1049-57.
36. Blais MA. Clinician ratings of the five-factor model of personality and the DSM-IV personality disorders. J Nerv Ment Dis. 1997:185(6):388-93.

37. Aluja A, Cuevas L, García LF, García O. Zuckerman's personality model predicts MCMI-III personality disorders. Pers Individ Dif. 2007a; 42 (7): 1311-1321.

38. Aluja A, Cuevas L, García LF. Predictions of the MCMI-III personality disorders from NEO-PI-R domains and facets: Comparison between American and Spanish samples. Int J Clin Health Psychol. 2007b; 7 (2): 307-321.

39. Trobst KK, Ayearst LE, Salekin RT. Where is the personality in personality disorder assessment? A comparison across four sets of personality disorder scales. Multivariate Behav Res. 2004;39(2):231-71.

40. Leible TL, Snell Jr WE. Borderline personality disorder and multiple aspects of emotional intelligence. Pers Individual Differ. 2004;37(2):393-404.

41. Trull TJ. DSM-III-R personality disorders and the five-factor model of personality: An empirical comparison. J Abnorm Psychol. 1992;101(3):553-60.

42. Ball SA, Tennen H, Poling JC, Kranzler HR, Rounsaville BJ. Personality, temperament, and character dimensions and the DSM-IV personality disorders in substance abusers. J Abnorm Psychol. 1997;106(4):545-53.

43. Wang W, Hu L, Mu L, Chen D, Song Q, Zhou M, et al. Functioning styles of personality disorders and five-factor normal personality traits: a correlation study in Chinese students. BMC Psychiatry. 2003;3:11.

44. Yu R, Yu S, Liu Y, Chen W, Shen M, Wang D, et al. Adjectival descriptors for antisocial personality trait in Chinese university students. J Pers Disord. 2009;23(6):661-8.

45. Wilson DS, Near D, Miller RR. Machiavellianism: a synthesis of the evolutionary and psychological literatures. Psychol Bull. 1996;119(2):285-99.

46. Lozano BE, Johnson SL. Can personality traits predict increases in manic and depressive symptoms? J Affect Disord. 2001;63(1-3):103-11.

47. Fineberg NA, Day GA, de Koenigswarter N, Reghunandanan S, Kolli S, Jefferies-Sewell K, et al. The neuropsychology of obsessive-compulsive personality disorder: a new analysis. CNS Spectr. 2015;20(05):490-9.

48. Samuel DB, Widiger TA. Conscientiousness and obsessive-compulsive personality disorder. Pers Disord Theory Res Treat. 2011;2(3):161-74.

49. Rector NA, Hood K, Richter MA, Bagby RM. Obsessive-compulsive disorder and the five-factor model of personality: distinction and overlap with major depressive disorder. Behav Res Ther. 2002:40(10):1205-19.

50. Paunonen SV, Zeidner M, Engvik HA, Oosterveld P, Maliphant R. The nonverbal assessment of personality in five cultures. J Cross Cult Psychol. 2000;31:220-39.

51. Gao Q, Ma G, Zhu Q, Fan H, Wang W. Predicting personality disorder functioning styles by the Five-Factor Nonverbal Personality Questionnaire in healthy volunteers and personality disorder patients. Psychopathology. 2016:49(1):5-12

52. Le Corff Y, Toupin J. The five-factor model of personality at the facet level: association with antisocial personality disorder symptoms and prediction of antisocial behavior. J Psychopathol Behav Assess. 2010;32:586-94.

53. Bradlee PM, Emmons RA. Locating narcissism within the interpersonal circumplex and the five-factor model. Person Indiv Diff. 1992;13:821-30.

\section{Submit your next manuscript to BioMed Central and we will help you at every step:}

- We accept pre-submission inquiries

- Our selector tool helps you to find the most relevant journal

- We provide round the clock customer support

- Convenient online submission

- Thorough peer review

- Inclusion in PubMed and all major indexing services

- Maximum visibility for your research

Submit your manuscript at www.biomedcentral.com/submit 